\section{Laser Iridotomy}

British Medical Fournal, 1970, 2, 580-581

$S^{\mathrm{ma}}$ ummary: A ruby laser has been used to produce a permeable lesion in the iris to establish a communication between the anterior and posterior chambers. In a preliminary study in nine patients the technique gave satisfactory results in the prophylactic treatment of four cases of incipient closed-angle glaucoma and of two cases of iris bombé following uveitis.

\section{INTRODUCTION}

Peripheral iridectomy is established as a very satisfactory operation in early cases of closed-angle glaucoma and for the prophylactic treatment of the second eye in patients who have suffered an acute attack. Its efficiency depends on the establishment of a low-resistance pathway for aqueous humour from the posterior to the anterior chamber, bypassing the normal route through the pupil and preventing so-called physiological iris bombé. Though it is not a technically difficult operation, complications do occur, and it cannot safely be performed as an outpatient procedure.

Extensive experience with animals has shown that it is possible to irradiate the iris with a pulsed laser without damage to the cornea, lens, or retina (Hallman et al., 1968, 1969). With a ruby laser having an output of about $200 \mathrm{~mJ}$ concentrated on an area $1 \mathrm{~mm}$. in diameter, a single exposure rarely causes a complete hole in the iris. The energy of the laser beam is only released where it is absorbed, and maximum absorption takes place in the heavily pigmented epithelium of the posterior surface of the iris. The looser stromal tissue is less heavily pigmented and therefore absorbs less energy, and it is not disrupted as completely as the pigment epithelium. Most probably, however, the latter structure is the layer of the iris least permeable to aqueous. If this layer is inadvertently left behind in attempting a peripheral iridectomy no communication is established between the posterior and anterior chambers.

The injection of fluorescein into the posterior chamber in rabbit eyes has shown that about 10 to 12 days after a single laser irradiation the fluorescein seeps freely through the area of the lesion. Destruction of the remaining iris stroma in this area can often be obtained by repeated exposures, but once the pigment epithelium is destroyed enough light energy can pass through the iris to produce a retinal lesion (Snyder, 1967).

\section{METHODS}

For clinical use a ruby laser (C. Davis Keeler) has been mounted on a modified Gambs photo-slit-lamp so that the laser beam is coincident with the illuminating system of the slit-lamp. This arrangement allows accurate placing of the lesion and continuous viewing through the microscope. The laser beam can be focused to a circular area $1 \mathrm{~mm}$. in diameter or defocused slightly to give a $2-\mathrm{mm}$. beam. In the latter case the energy is incrased to give about the same energy density, $18 \mathrm{~J} / \mathrm{sq}$. cm. It is important to transilluminate the iris (by the method described by Abrams (1964)) to ensure that the pigment epithelium is intact in the area to be lasered.

No anaesthesia is required, but the pupil is contracted with drops of pilocarpine $2 \%$ if it is not already miotic. One drop of prednisolone disodium phosphate (Predsol) is instilled every five minutes for half an hour before lasering and continued at half-hourly intervals for two hours afterwards. The patient is positioned at the slit-lamp, the beam being aimed obliquely at the periphery of the upper part of the iris. Aiming obliquely ensures that even if sufficient energy traversed the iris to cause a retinal lesion, only a peripheral part of the retina could be affected. After exposure the iris is again transilluminated to confirm destruction of the pigment epithelium. The patient is kept under observation and tension readings are recorded over the next three to four hours.

\section{Results}

Nine patients have so far been treated-prophylactic operations in four, established closed-angle glaucoma in two, iris bombé following uveitis in two, and optical iridotomy in one.

\section{Prophylactic Operations}

All four patients had had an acute attack of closed-angle glaucoma in the other eye. In three of the eyes treated prophylactically a satisfactory area of transillumination was produced in the periphery of the iris after one exposure; particles of pigment could be seen flowing in a stream through the pupil. No immediate change in the width of the angle was observed, but after one to two weeks there was a definite increase in width.

The fourth patient was an Indian with a deeply pigmented iris and trachomatous scarring of the cornea. As no transillumination could be seen follcwing a single exposure, treatment was repeated to the same area one week later. Some disruption of the anterior surface of the iris occurred and a stream of pigment particles could be seen emerging through the pupil. Transillumination was difficult to observe because of the corneal scarring, but one week after the second exposure gonioscopy showed that the angle was wider than before and seemed unlikely to close.

Though the follow-up period (up to three months) is too short for final evaluation, the procedure seems to have achieved the object of reducing iris bombe and opening the angle in these cases. In one case the dark-room test, which had been positive, was negative two months after treatment. The regimen of local steroid therapy before and after lasering was used in all four cases, and in none of them was there any evidence of inflammatory reaction, pain, or discomfort.

\section{Established Closed-Angle Glaucoma}

The first of the two patients had been admitted to hospital with an acute attack of closed-angle glaucoma one week previously. The ocular tension had decreased to $22 \mathrm{~mm}$. $\mathrm{Hg}$ with medical treatment. The cornea was still oedematous and the angle very narrow. The iris was dark brown in colour, and laser irradiation produced slight pallor of the surface and pigment scatter surrounding the lesion. No transillumination could be seen. A second exposure five days later produced more disruption of the surface of the iris but no transillumination. As the nasal half of the angle still appeared to be closed and the tension was not adequately controlled, a peripheral iridectomy was performed. Unfortunately the lens was damaged and subsequently became swollen, with the development of malignant glaucoma. By this time, three weeks after the second laser application, the laser lesion showed transillumination, but the raised tension necessitated extraction of the lens and a broad iridectomy. It is probable that laser iridotomy was unsuccessful in this case because of persistent angle block. 
The second patient had a four-year history of recurrent attacks of anterior uveitis in the right eye. She then developed raised tension in this eye, which had a shallow anterior chamber. The dark-room test was positive and the tension over $25 \mathrm{~mm}$. $\mathrm{Hg}$ even with miotic treatment. Two laser lesions were produced at 12 o'clock, resulting in immediate transillumination. The tension rose after the treatment and remained above $40 \mathrm{~mm}$. $\mathrm{Hg}$ for three days; treatment with acetazolamide (Diamox) and pilocarpine reduced it to below $30 \mathrm{~mm}$. $\mathrm{Hg}$, but gonioscopy showed that much of the angle was probably closed. She was admitted to hospital and a peripheral iridectomy performed. In spite of the production of a drainage bleb she still required miotics to control the tension after the operation. As in the previous case, it is probable that permanent damage had already occurred in the angle before the laser irradiation. Steroids before and after lasering were not used in this patient.

\section{IRIS BombE Following UveITIS}

These two patients had a long history of anterior uveitis with almost complete occlusion of the pupil, posterior synechiae, and the development of iris bombé. In both patients transillumination of the iris was seen immediately after exposure. In one patient the tension rose during the subsequent three days and the iris appeared oedematous with dilatation of the iris vessels. The tension was controlled with acetazolamide and local steroids. One month later the iris bombé was much reduced; the eye was quiet, and has remained so, with a normal tension, for another 15 months.

The other patient showed a definite improvement in the iris bombé one week after treatment. Gonioscopy showed the angle to be more open, but also revealed some anterior synechiae. The tension has remained rather variable in both eyes though the angles are open, and it is probable that the long-standing uveitis has caused some permanent damage to the angles. The iris bombé has not recurred during a followup period of 14 months.

\section{OPTICAL IRIDOTOMY}

This patient had developed severe uveitis and secondary cataract after an operation for glaucoma. Subsequent cataract operation was followed by a drawn-up pupil occluded by a cyclitic membrane. Laser irradiation was tried in the hope of forming an artificial pupil. A hole was produced from one exposure, but revealed a dense white cyclitic membrane behind the iris.

\section{COMPLiCATIONS}

No evidence of damage was observed in the cornea, lens, or fundus, and no haemorrhage occurred from iris vessels. The two eyes which reacted to lasering with a rise of tension had had previous attacks of anterior uveitis and were among those treated before local steroids were used routinely before and after lasering.

\section{Comment}

Laser irradiation of the human iris seems to be a safe procedure which produces destruction of the pigment epithelium and the subsequent formation of an area of iris permeable to aqueous humour. It is painless, does not require admission to hospital, and does not prejudice the success of any subsequent operative procedure. A single exposure is adequate in a light-coloured iris, but a dark iris may require more than one exposure. This is likely to be most useful as a prophylactic procedure and for the treatment of iris bombé following inflammatory occlusion of the pupil.

I am grateful to the surgeons of Moorfields Eye Hospital and to Mr. M. B. R. Mathalone, of the Royal Eye Hospital, for referring cases, and to Mr. Martin Bass for his skilled technical assistance. This work was made possible by a grant from the Wates Foundation.

E. S. PERKINS, M.D., PH.D., F.R.C.S., Professor of Experimental Ophthalmology,

Institute of Ophthalmology, London W.C.1. University of London.

\section{REFERENCES}

Abrams, J. D. (1964). British fournal of Ophthalmology, 48, 42.

Hallman, V. L., Perkins, E. S., Watts, G. K., and Wheeler, C. B. (1968), Experimental Eye Research, 7, 481.

Hallman, V. L., Perkins, E. S., Watts, G. K., and Wheeler, C. B. (1969). Experimental Eye Research, 8, 1.

Snyder, W. B. (1967). Archives of Ophthalmology, 77, 93.

\section{Medical Memoranda}

\section{Thrombocytopenic Purpura after Blood Transfusion}

\section{British Medical fournal, 1970, 2, 581-582}

Morrison and Mollison (1966) described a patient who developed thrombocytopenic purpura six days after blood transfusion and reviewed five similar cases from the literature. In these patients-all of whom were parous women-a platelet isoantibody, termed anti-P1 al by Shulman et al. (1961), was found. We report a case of thrombocytopenic purpura occurring after blood transfusion in a nulliparous woman of 68 with a retroperitoneal liposarcoma.

\section{CASE REPORT}

The patient was admitted to the London Hospital on 10 June, 1968 with a history of an abdominal mass and dysphagia for eight months and loss of weight and symptoms of anaemia for one month. There was no history of blocd transfusion, pregnancy, spontaneous bleeding, purpura, or allergy. Dichloralphenazone, given as a night sedative, was the only drug used before operation.

On examination she was pale and there was a large irregular abdominal mass extending from the left costal margin to the left iliac fossa. Barium meal examination showed a filling defect of the greater curvature thought to be due to an extrinsic mass. Chest $x$-ray examination was normal. The haemoglobin was $8.2 \mathrm{~g} . / 100 \mathrm{ml}$. The mean corpuscular haemoglobin concentration was $27 \%$, and the film showed a slight hypochromia with normal leucocytes and platelets. She was blood group A Rh-positive. Three units of packed bank blood were transfused (14 June) and the haemoglobin rose to $11.6 \mathrm{~g} . / 100 \mathrm{ml}$. At laparotomy (17 June) a large pedunculated retroperitoneal liposarcoma weighing $7 \mathrm{lb} .12 \mathrm{oz}$. $(3,500$ g.) was removed. During the operation she received four units of blood. The immediate postoperative course was uneventful.

On the third postoperative day her nose started bleeding; this became continuous. A large bruise appeared on her right arm. The Hess test was strongly positive and the platelet count was less than $10,000 /$ cu.mm. She was transfused with fresh whole blood. On the next day purpura had become widespread, haematuria 